\title{
Chronic phospholamban inhibition prevents progressive cardiac dysfunction and pathological remodeling after infarction in rats
}

\author{
Yoshitaka Iwanaga,1,2 Masahiko Hoshijima,1,2 Yusu Gu,1,2 Mitsuo Iwatate,1,2 Thomas Dieterle,1,2 \\ Yasuhiro Ikeda, ${ }^{1,2,3}$ Moto-o Date, ${ }^{1,2}$ Jacqueline Chrast,,1,2 Masunori Matsuzaki, ${ }^{3}$ Kirk L. Peterson,, ${ }^{1,2}$ \\ Kenneth R. Chien, ${ }^{1,2}$ and John Ross, Jr.1,2
}

${ }^{1}$ Department of Medicine, and ${ }^{2}$ Institute of Molecular Medicine, University of California, San Diego, La Jolla, California, USA. ${ }^{3}$ Department of Medical Bioregulation, Yamaguchi University Graduate School of Medicine, Yamaguchi, Japan.

\begin{abstract}
Ablation or inhibition of phospholamban (PLN) has favorable effects in several genetic murine dilated cardiomyopathies, and we showed previously that a pseudophosphorylated form of PLN mutant (S16EPLN) successfully prevented progressive heart failure in cardiomyopathic hamsters. In this study, the effects of PLN inhibition were examined in rats with heart failure after myocardial infarction (MI), a model of acquired disease. S16EPLN was delivered into failing hearts 5 weeks after MI by transcoronary gene transfer using a recombinant adeno-associated virus ( $\mathrm{rAAV}$ ) vector. In treated (MI-S16EPLN, $n=16$ ) and control (MI-saline, $n=18$ ) groups, infarct sizes were closely matched and the left ventricle was similarly depressed and dilated before gene transfer. At 2 and 6 months after gene transfer, MI-S16EPLN rats showed an increase in left ventricular (LV) ejection fraction and a much smaller rise in $L V$ end-diastolic volume, compared with progressive deterioration of $L V$ size and function in MI-saline rats. Hemodynamic measurements at 6 months showed lower LV end-diastolic pressures, with enhanced LV function (contractility and relaxation), lowered LV mass and myocyte size, and less fibrosis in MI-S16EPLN rats. Thus, PLN inhibition by in vivo rAAV gene transfer is an effective strategy for the chronic treatment of an acquired form of established heart failure.
\end{abstract}

\section{Introduction}

Advances in medical and surgical treatments have substantially reduced the mortality from cardiac disease, but chronic heart failure primarily of ischemic origin remains a leading cause of morbidity and mortality (1). Given the limits of cardiac transplantation (2), new forms of treatment are needed that can target the underlying biological processes within the cardiomyocyte that lead to chronic dysfunction and unfavorable remodeling $(3,4)$. Gene therapy has been proposed as one such promising strategy. However, there have been several limitations to the application of gene transfer for clinical and experimental heart failure, including lack of a gene-delivery system that can provide high efficiency and cardiac specificity, and the need for vectors that give stable long-term expression (5). Recently, we developed a novel in vivo transcoronary gene-delivery system that achieved high efficiency in myocardium when applied in the cardiomyopathic hamster using an adenovirus vector (6), and relatively cardiac-specific, long-term expression was then achieved with a recombinant adeno-associated virus (rAAV) vector in that hamster model (7).

Failing heart muscle generally exhibits distinct changes in intracellular $\mathrm{Ca}^{2+}$ handling, including impaired removal of cytosolic $\mathrm{Ca}^{2+}$;

\footnotetext{
Nonstandard abbreviations used: calcium/calmodulin-dependent kinase II (CaMKII); early mitral orifice inflow velocity (E); inflow velocity during atrial contraction (A); left ventricular (LV); LV ejection fraction (LVEF); LV end-diastolic pressure (LVEDP); LV end-diastolic volume (LVEDV); LV end-systolic volume (LVESV); muscle-specific Lim protein (MLP); myocardial infarction (MI); phospholamban (PLN); recombinant adeno-associated virus (rAAV); sarcoplasmic reticulum (SR);

$\mathrm{SR} \mathrm{Ca}^{2+}$-ATPase 2 (SERCA2).

Conflict of interest: Kenneth Chien and Masahiko Hoshijima have a financial interest in these studies.

Citation for this article: J. Clin. Invest. 113:727-736 (2004). doi:10.1172/JCI200418716
}

reduced $\mathrm{Ca}^{2+}$ loading of the cardiac sarcoplasmic reticulum (SR) with downregulation of $\mathrm{SR} \mathrm{Ca}^{2+}$-ATPase 2 (SERCA2); and defects in $\mathrm{SR} \mathrm{Ca}^{2+}$ release accompanied by impairment of cardiac relaxation and systolic function $(8,9)$. However, therapeutic attempts to target these molecular abnormalities for the treatment of experimental heart failure using in vivo gene transfer strategies were limited to short-term overexpression of SERCA2 (10) or overexpression of upstream regulators of $\mathrm{Ca}^{2+}$ handling, such as a $\beta$-adrenergic receptor kinase-inhibitory peptide (11) using adenovirus vectors.

Initially, we crossed mice with ablated phospholamban (PLN), an endogenous inhibitor of SERCA2, with a muscle-specific Lim protein-null (MLP-null) mouse model of dilated cardiomyopathy and demonstrated complete prevention of the heart failure phenotype in the offspring, along with improved cardiac $\mathrm{SR} \mathrm{Ca}^{2+}$ storage and release (12). Subsequently a PLN-null mutation was reported to prevent systolic dysfunction and exercise intolerance, but not hypertrophy, in a murine model of hypertrophic cardiomyopathy (13). Later, we generated a pseudophosphorylated mutant PLN peptide, S16EPLN, designed to mimic the conformational changes in PLN following its phosphorylation by cAMPdependent protein kinase, and when the S16EPLN gene was delivered into BIO14.6 cardiomyopathic hamsters, the usually rapid progression of cardiac dysfunction over the 7-month study period was substantially alleviated (7).

Recent studies in transgenic models, however, have not supported a therapeutic effect of PLN ablation on cardiac hypertrophy and dysfunction. In one study, apoptotic heart failure resulted from overexpression of a signaling molecule, or severe neonatal-onset cardiac dilation was induced by targeted mutation of a structural protein, and neither was modified by PLN ablation (14). Also, Kiriazis et al. have reported that hyperdynamic PLN-null mice do not differ 
from wild-type mice in the development of hypertrophy or in the incidence of heart failure after sustained surgical aortic stenosis (15).

In addition, two recent clinical studies have suggested the possibility that interference with the PLN-SERCA2 interaction may be detrimental. Two types of human PLN mutations (R9C and L39stop) were linked to human dilated cardiomyopathy, and distinctive mechanisms were proposed for each mutation to induce cardiac defects by altering SR $\mathrm{Ca}^{2+}$ handling $(16,17)$.

Thus, it is critical to evaluate the role of PLN inhibition in a model of acquired heart failure that has proven fidelity and predictability for therapeutic effects in human disease. The progressive heart failure that occurs after myocardial infarction (MI) in the rat is a validated and widely accepted model of the most common form of heart failure observed in humans. In the present study, we successfully applied a modified transcoronary gene-delivery method in chronically failing hearts of rats after MI. The goal of the study was to determine whether sustained action of the S16EPLN protein in this model could affect global left ventricular (LV) function and remodeling in the setting of established heart failure.

\section{Methods}

Experiments were carried out in adult male Sprague-Dawley rats obtained from Charles River Laboratories Inc. (Wilmington, Massachusetts, USA). The animals were housed at $20^{\circ} \mathrm{C} \pm 2{ }^{\circ} \mathrm{C}, 55 \% \pm 20 \%$ humidity, with 12-hour light/dark cycles and free access to food and water in the Animal Care Facility at the University of California, San Diego. The study was approved by the University of California, San Diego Animal Subjects Committee.

Construction of recombinant viral vectors. $\mathrm{rAAV}$ vectors carrying S16EPLN (rAAV-S16EPLN) and rAAV vectors carrying a LacZ marker gene (rAAV-LacZ) were constructed as previously described (7) and produced by Lili Wang and James Wilson at the University of Pennsylvania (Philadelphia, Pennsylvania, USA). In brief, rAAV type 2 vectors were made by the three-plasmid cotransfection method (18) and recovered by a single-step heparin-column method (19). The genome titer (genome copy) was determined by real-time PCR. Recombinant adenovirus type 5 vectors with the LacZ gene (AdenoLacZ) and with the S16EPLN gene (Adeno-S16EPLN) were prepared as previously described (6).

Generation of MI rats. When the rats were 7-8 weeks old, left coronary artery ligation was performed as previously described (20). Briefly, rats were anesthetized with ketamine $(100 \mathrm{mg} / \mathrm{kg})$ and xylazine $(10 \mathrm{mg} / \mathrm{kg})$, endotracheally intubated, and mechanically ventilated with room air (respiratory rate 55-65 breaths per minute, tidal volume $2.5 \mathrm{ml}$ ). Under sterile conditions, a left anterior thoracotomy was performed to expose the heart. The left anterior descending coronary artery was ligated between the right ventricular outflow tract and the left atrium with a 6-0 silk suture that was passed through the superficial layers of myocardium (20). The lungs were then reinflated and the chest incision closed. Sham-operated rats (no-MI rats) were prepared in the same manner but did not undergo coronary artery ligation.

Randomization of MI rats for gene transfer. At 5 weeks after coronary ligation, following echocardiographic determination of cardiac function and MI size, animals with an infarct size between $30 \%$ and $40 \%$ were randomized into two groups: rAAV-S16E gene transfer (MI-S16EPLN) and saline injection (MI-saline). In the MIsaline group, a volume of saline alone equal to that of the virus solution was injected with the same procedures for gene transfer used in the MI-S16EPLN group.
Echocardiography. Echocardiograms to assess LV size and function were recorded as previously described (21) at 5 weeks after coronary occlusion (before randomization) and at 2 months and 6 months after the gene transfer procedure. Light anesthesia was induced with intraperitoneal ketamine $(50 \mathrm{mg} / \mathrm{kg})$ and xylazine $(5 \mathrm{mg} / \mathrm{kg})$. Twodimensional parasternal long-axis images were obtained perpendicular to the short axis and were deemed appropriate at the site where LV length was maximum and both mitral and aortic valves were contained in the image. The LV endocardial border was manually traced, and long-axis dimensions and LV areas at end-systole and end-diastole were determined. The LV end-diastolic volume (LVEDV), LV end-systolic volume (LVESV), and LV ejection fraction (LVEF) were calculated using the area-length method, which has been validated in rodents and humans (22). Three investigators blinded as to experimental groups agreed on the optimal echo frame for visualizing the left ventricle in the two-dimensional long-axis view at each time point. The frames selected were then analyzed in a random, blinded manner by two experienced investigators. The means of the differences between these two investigators were $0.001 \pm 0.047 \mathrm{ml}( \pm \mathrm{SD})$ for LVEDV, $0.005 \pm 0.032 \mathrm{ml}$ for LVESV, and $0.297 \pm 2.27 \%$ for LVEF. After completion of two-dimensional imaging, pulse-wave Doppler interrogation of mitral flow was performed in a parasternal longaxis view in order to evaluate diastolic filling; the ratio of early mitral orifice inflow velocity $(\mathrm{E})$ to inflow velocity during atrial contraction (A) was calculated (23).

In this study, in which treatment was expected to alter $L V$ remodeling, it was necessary to have a reliable estimate of the extent of the completed MI relative to the LV size prior to randomization into treated and untreated groups. Therefore an initial study was done in a separate group of ten rats, comparing echocardiographic and postmortem histological methods. Myocardial infarct size was estimated by the centerline method (24) (which measures wall excursion at 100 points around the LV circumference) in two-dimensional short-axis images at three LV levels (mid-papillary muscle, chordae tendineae, and near the apex), and the size estimates at the three levels were averaged to determine the percentage of the LV circumference that demonstrated systolic akinesis or dyskinesis. Values obtained by this method were compared with those obtained by histological quantification (25) at these three regions; a highly significant correlation was found $\left(r^{2}=0.948, P<0.01\right)$, and the mean of the differences in infarct size between the two methods was $0.10 \pm 2.98 \%( \pm \mathrm{SD})$.

Gene transfer. The gene transfer procedure in normal and MI rats was developed through modifications of our hamster gene transfer protocol (6). Briefly, animals were anesthetized with sodium pentobarbital $(70 \mathrm{mg} / \mathrm{kg}$ intraperitoneally), intubated, and ventilated. A small left anterior thoracotomy was performed, and string occluders were placed loosely around the ascending aorta and main pulmonary artery. A PE-60 catheter (Becton Dickinson and Co., Sparks, Maryland, USA) was then inserted through the right carotid artery into the aortic root just above the aortic valve and below the occluder for measurement of pressure and for injections, and the animals were then subjected to general hypothermia by external cooling with ice packs to below $26^{\circ} \mathrm{C}$. The great vessels (pulmonary artery followed by aorta) were then occluded. Next, $0.4 \mathrm{ml}$ of cardioplegic solution (6) was injected through the aortic catheter, followed by 0.4 $\mathrm{ml}$ of cardioplegic solution containing $10 \mu \mathrm{g}$ of substance $\mathrm{P}(26)$, and, after 60 seconds, $2.0 \mathrm{ml}$ of cardioplegic solution containing 5 $\mu \mathrm{g}$ of substance $\mathrm{P}$ and the viral vector was delivered. The occlusions were released 90 seconds later, dobutamine was administered, and the animals were rewarmed. 
In preliminary studies, Adeno-LacZ with $1.7 \times 10^{9}$ to $4.0 \times 10^{9}$ virus particles per gram body weight and rAAV-LacZ with $2.0 \times 10^{9}$ genome copies per gram body weight were evaluated for LacZ marker gene expression at 4 days and 5 weeks, respectively, after gene transfer by $\beta$-gal staining performed in three transverse cardiac sections per animal. The percentage of $\beta$-gal-positive myocytes was determined as previously reported in our laboratory (6). During these preliminary studies, the optimal volume of the solution containing cardioplegic solution, substance $\mathrm{P}$, and viral vector was assessed. The highest efficiency was achieved with the largest volume tested, $2.0 \mathrm{ml}$, which was then used in the randomized studies in which rAAV-S16EPLN $\left(1.9 \times 10^{9}\right.$ genome copies per gram body weight) or saline was administered in the MI rats.

Hemodynamic measurements. At 6 months after gene transfer, immediately after the final echocardiographic study, hemodynamic measurements were performed. A 2-French catheter-tip pressure transducer catheter (SPR-407; Millar Instruments Inc., Houston, Texas, USA) was introduced into the left ventricle through the right carotid artery (proximal to the site of prior catheter insertion for gene transfer) for measurements of LV pressures, and contractility and relaxation were assessed by maximum and minimum first derivatives of $L V$ pressure ( $\mathrm{LV} \mathrm{d} P / \mathrm{d} t$ ) and $\tau$, the time constant of relaxation (using an exponential function), as previously described (21). After base-line measurements, each animal underwent stepwise dobutamine infusions $(0.75-4 \mu \mathrm{g} / \mathrm{kg} / \mathrm{min})$ with data recordings at steady state at each dose.

Meridional stress of the LV wall at peak-systole and end-diastole was estimated, using non-simultaneous data, as $P R_{i} \div 2 h\left(1+b / 2 R_{i}\right)$, where $P$ is LV pressure, $R_{i}$ is inner LV minor-axis radius, and $b$ is the mean of anterior and posterior wall thickness values (21).

Histological analysis. For morphometric analysis, LV specimens were fixed with $10 \%$ buffered formalin and embedded in paraffin. Several sections were prepared from each specimen and stained with $\mathrm{H} \& \mathrm{E}$ for assessment of myocardial cellular infiltrates and inflammation, and with Masson's trichrome stain to assess the area of fibrosis using a point-counting method (27). Myocyte diameters were measured as previously described (28). In brief, in the sections stained with H\&E, a total of 100 myocardial cells were randomly selected from the noninfarcted area from each animal, and the shortest diameters of transversely cut fibers were measured at the level of the nucleus with the aid of an image analyzer (LUZEX 3U; Nikon Corp., Tokyo, Japan). This method was used to minimize errors in comparing different tissue sections for estimation of the size of cardiomyocytes, since slight offset can occur in transverse sectioning.

Western blot analysis. SR microvesicles were isolated from the noninfarcted posterior-lateral region of the left ventricle by sequential centrifugation as previously described (29). Protein concentration was determined by the bicinchoninic acid assay (Pierce Chemical Co., Rockford, Illinois, USA) using BSA as standard. Twenty micrograms of protein was electrophoresed on 4-12\% polyacrylamide gels and transferred to PVDF membranes (Bio-Rad Laboratories Inc., Hercules, California, USA). Membranes were incubated with primary antibodies for 1 hour at room temperature and then with appropriate HRP-conjugated secondary antibodies. The immune complexes were detected with a chemiluminescence kit (Pierce Chemical Co.). The primary antibodies were anti-PLN (clone A1; Upstate Biotechnology Inc., Lake Placid, New York, USA), anti-SERCA2 (Affinity BioReagents Inc., Golden, Colorado, USA), and anti-phospho-PLN (Ser16) (Upstate Biotechnology Inc.).
The endogenous wild-type PLN and the S16EPLN transgene were measured using in vitro phosphorylation followed by immunoblotting, as previously described (7). Briefly, the protein samples were treated with protein kinase A to saturate the level of phosphorylation reaction and then subjected to immunoblotting. An anti-phospho-PLN (Ser16) antibody was used to measure the endogenous wild-type PLN that could be phosphorylated. It should be noted that, although S16EPLN was designed as a pseudophosphorylated PLN mutant, it is not detectable with the anti-phospho-PLN (Ser16) antibody. On the other hand, the anti-PLN antibody used in this study recognizes both S16EPLN and the wild-type PLN with equivalent sensitivities and was used to determine the sum of the S16EPLN transgene and the endogenous wild-type PLN. The level of the S16EPLN was estimated by the difference between that total amount of PLN and the level of PLN that was phosphorylated to the level of saturation by protein kinase A.

Demonstration of S16EPLN effectiveness in rat cardiomyocytes. To establish that the rat heart responds to S16EPLN in a manner similar to
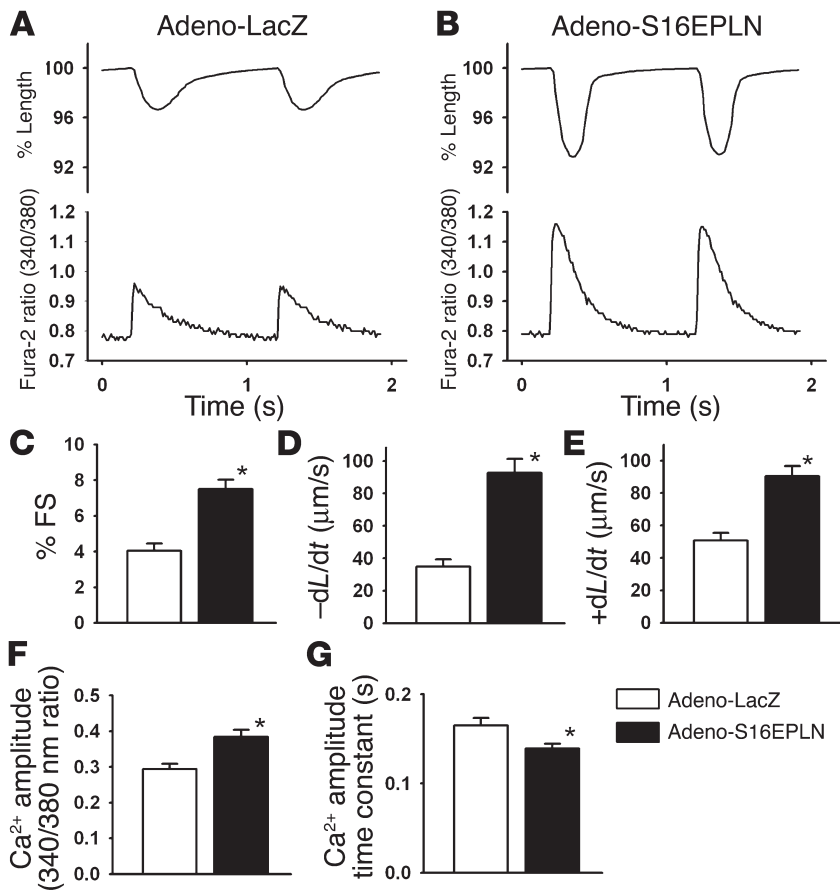

\section{Figure 1}

Enhanced shortening and SR calcium cycling in isolated adult rat cardiomyocytes treated with S16EPLN. Ventricular cardiomyocytes were isolated from adult Wistar rats according to the protocol described by Zho et al. (30). Cells were transfected with adenovirus vectors expressing LacZ (Adeno-LacZ) or S16EPLN (Adeno-S16EPLN) at a multiplicity of infection of 100 and cultured for 36 hours. Nearly $100 \%$ efficiency of transfection was confirmed by $\beta$-gal staining of Adeno-LacZ-treated cells. Thereafter, both Adeno-LacZ-treated cells and Adeno-S16EPLN-treated cells were loaded with fura-2/AM, and cell shortening and changes in intracellularcalcium concentration were monitored. (A and $\mathbf{B}$ ) Representative tracings of cell length and fura-2 340/380 ratio, an index of calcium concentration. (C-E) Indices of cell shortening (percentage fractional shortening [\% FS] and $-\mathrm{d} L / \mathrm{d} t)$ and relaxation $(+\mathrm{d} L / \mathrm{d} t)$. (F and $\mathbf{G})$ The averaged peak amplitude of an index of calcium transient (F) and the averaged decay time constant of the descending limb of calcium transient (G). Data represent mean \pm SE and are accumulated from five independent experiments from five animals. A total of 40 cells were measured for each treatment group. ${ }^{*} P<0.05$ between groups. $L$, length. 

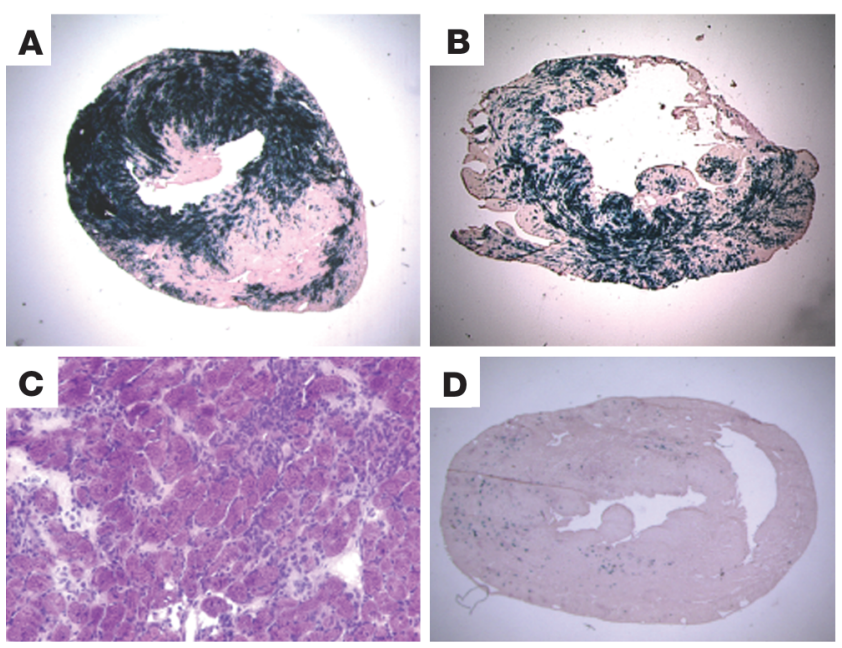

that of other species, an adenovirus vector carrying S16EPLN was transfected in isolated normal adult rat cardiomyocytes for 36 hours (30), and cell contraction and $\mathrm{Ca}^{2+}$ transients were measured using an edge-motion detector and a dual-excitation spectrofluorometer (IonOptix Corp., Milton, Massachusetts, USA) as previously described (31). An enhanced peak $\mathrm{Ca}^{2+}$ transient in systole and a decrease in the time constant of $\mathrm{Ca}^{2+}$-transient decay $(\tau)$ were noted accompanying clearly enhanced cell shortening and relaxation (Figure 1).

In addition, the same adenovirus vector was transferred in vivo in normal rats using the transcoronary gene transfer procedure. Four days after gene transfer, the animals were subjected to echocardiography. Increased percentage fractional shortening (S16EPLN-treated, $47.2 \pm 0.6$; saline-treated, $39.0 \pm 1.4 ; n=6$ each; $P<0.05)$ and slightly smaller LV diastolic chamber size (LV end-diastolic diameter: S16EPLN-treated, $7.40 \pm 0.12 \mathrm{~mm}$; saline-treated, $8.02 \pm 0.16$ $\mathrm{mm} ; n=6$ each; $P<0.05)$ were observed with short-term S16EPLN treatment. Hemodynamic analysis of the same animals showed a trend toward augmentation of positive maximum $L V \mathrm{~d} P / \mathrm{d} t$ by S16EPLN treatment (S16EPLN-treated, 7,069 $\pm 456 \mathrm{mmHg} / \mathrm{s}$; saline-treated, 6,260 $\pm 299 \mathrm{mmHg} / \mathrm{s} ; n=6$ each), which did not reach statistical significance $(P=0.164)$.

Statistical analysis. All values are expressed as mean \pm SE unless specified otherwise. The significance of the differences between group means for most data was analyzed by one-way ANOVA with post hoc comparisons by the Student-Newman-Keuls test. For serial echocardiographic data, the main effects of treatment were tested using twofactor ANOVA for repeated measures, and differences between the groups at specific time points were assessed using two-factor ANOVA with post hoc comparisons by the Student-Newman-Keuls test. A value of $P<0.05$ was considered statistically significant.

\section{Results}

Establishment of in vivo high-efficiency gene delivery in MI rats. Representative images stained for $\beta$-gal activity of normal and MI rat hearts are shown in Figure 2. Overall, in initial studies using Adeno-LacZ, the transduction efficiency was $55.3 \% \pm 2.3 \%$ (range, $33.2-81.7 \%$ of LV) in seven normal rats (Figure 2A) and $40.1 \% \pm 2.3 \%$ (range, $23.2-62.5 \%$ of $\mathrm{LV}$ ) in the noninfarcted regions of the heart in nine MI rats (Figure 2B). A range of viral-injectate volumes was studied, and since the highest efficiency (61.2\%) was associated with the highest volume of injectate $(2.0 \mathrm{ml})$ in $\mathrm{MI}$ rats, this volume was used in subsequent studies.

\section{Figure 2}

Representative images of $\beta$-gal activity in normal and MI rat LV slices after LacZ marker gene transfer. (A and $\mathbf{B}$ ) High-efficiency cardiac gene transfer is shown in normal rats $(\mathbf{A})$ and $\mathrm{MI}$ rats $(\mathbf{B})$ at 4 days after AdenoLacZ transfer. (C and D) Significant inflammatory responses are evident in $\mathrm{MI}$ rats at 5 weeks after rAAV-LacZ transfer. (C) H\&E staining. (D) $\beta$-gal staining. Magnifications: A, B, and D, $\times 4.5$; C, $\times 200$.

At 5 weeks after the transfection of rAAV-LacZ $(n=6)$, heavy inflammation with diffuse monocytic infiltration was observed in the LV of normal and MI rats (Figure 2C), and less than $1 \%$ of myocytes were positively stained for $\beta$-gal activity (Figure 2D). In contrast, inflammatory changes were not observed in rats that received the rAAV-S16EPLN vector over 6 months (data not shown).

Pre-gene transfer development of heart failure in MI rats. The coronary artery was ligated in 113 rats, of which 40 died in the first 48 hours (early mortality 35.4\%) and two died within 5 weeks (late mortality 1.8\%). Among the 71 survivors at 5 weeks after MI, the infarct size assessed by echocardiography was 30-40\% in 34 rats, and these animals were randomized into two groups (MI-S16EPLN and MI-saline) prior to the gene transfer procedure; thus, 22 rats with small $(<30 \%)$ infarctions and 15 rats with very large (>40\%) infarctions were excluded from the study. A group of age-matched sham-operated (no-MI) rats was also studied $(n=13)$. In all randomized MI rats, LV function was significantly depressed and LVEDV increased compared with no-MI rats, and there were no significant differences in LV size and function, or in infarct sizes, between the two randomized treatment groups prior to treatment (Table 1). In the subsequent gene transfer procedure, perioperative mortality (within 24 hours after surgery) was $14.7 \%$, with 13 survivors in the MI-S16EPLN group and 16 survivors in the MI-saline group.

Improvement of cardiac function, contractility, and suppression of global remodeling by $r A A V$-S16EPLN treatment in rats with post-MI heart failure. Four rats (19\%) in the MI-saline group died with signs of heart failure

\section{Table 1}

Base-line echocardiographic characteristics of MI rats before gene transfer

\begin{tabular}{lccc}
\hline & \multicolumn{3}{c}{ After MI (5 weeks) } \\
& No-MI & MI-saline & MI-S16EPLN \\
Number & 13 & $16(12)^{\mathrm{A}}$ & $13(12)^{\mathrm{A}}$ \\
Body weight (g) & $428 \pm 12$ & $408 \pm 8$ & $415 \pm 8$ \\
Infarct size (\%) & - & $37.1 \pm 0.65$ & $36.7 \pm 0.73$ \\
Heart rate (bpm) & $279 \pm 6$ & $292 \pm 6$ & $279 \pm 4$ \\
LVEF (\%) & $53.1 \pm 1.0$ & $32.4 \pm 0.5^{\mathrm{B}}$ & $32.4 \pm 1.0^{\mathrm{B}}$ \\
LVEDV (ml) & $0.68 \pm 0.03$ & $1.06 \pm 0.03^{\mathrm{B}}$ & $1.07 \pm 0.04^{\mathrm{B}}$ \\
& & & \\
\hline
\end{tabular}

Almmediate survivor (survivor 6 months after gene transfer). ${ }^{\mathrm{B} P}<0.05$ vs. no-MI. bpm, beats per minute. 

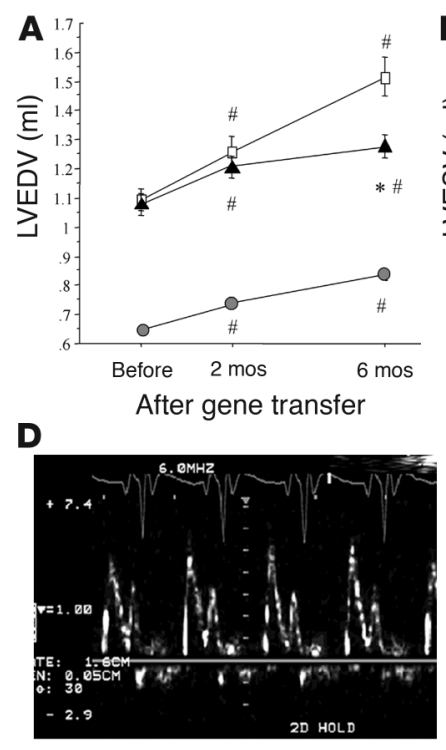

\section{C}

E

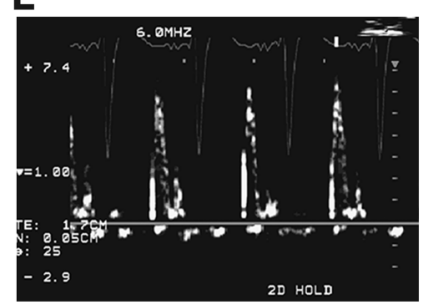

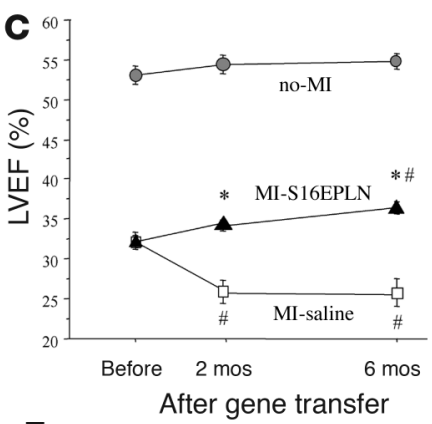

$\mathbf{F}$

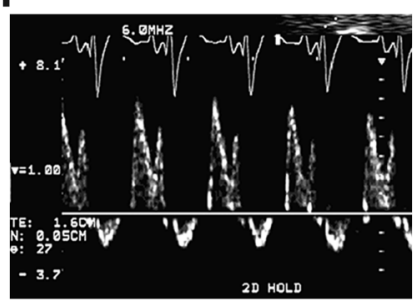

Figure 3

Serial changes of echocardiographic variables before and after S16EPLN gene transfer. (A) LVEDV. (B) LVESV. (C) LVEF. The S16EPLN treatment enhanced LV contractility and suppressed LV dilation over 6 months. Values are mean \pm SE. ${ }^{*} P<0.05$, MI-S16EPLN vs. MI-saline animals. ${ }^{\#} P<0.05$ vs. before gene transfer. Circles, no-Ml; squares, MI-saline; triangles, MI-S16EPLN. (D-F) Representative images of pulse-wave Doppler recordings of mitral flow at 6 months in no-Ml (D), MI-saline (E), and $\mathrm{MI}$ S16EPLN rats $(\mathbf{F})$. (e.g., peripheral edema, respiratory distress, and general fatigue), one at 2 months, one at 2.5 months, and two at 5.5 months after gene transfer, whereas one rat (7.7\%) in the MI-S16EPLN group died at 4 months of an unknown cause, without signs of heart failure. There was no death in the no-MI group.

By serial echocardiography in MI-saline rats, the LVEF progressively decreased (by 20.0\%) from pre-gene transfer base line, and LVEDV increased substantially (by 38.4\%) during the 6-month observation period (Figure 3, A and C). In no-MI rats, the LVEF did not change and the LVEDV increased moderately, paralleling natural growth. In contrast, the rAAV-S16EPLN treatment increased LVEF by $6.0 \%$ at 2 months and by $12.8 \%$ at 6 months compared with base line (Figure 3C). The LVEDV increased somewhat in the MIS16EPLN animals; however, the extent of change (by $18.2 \%$ at 6 months after gene transfer compared with pre-gene transfer base line) was $53.4 \%$ less than that occurring in the MI-saline group and was comparable to that in the no-MI animals (Figure 3A).

Improvement of diastolic LV function was shown in the MIS16EPLN versus the MI-saline group using pulse-wave Doppler recordings of mitral inflow. Increased early filling velocity $(\mathrm{E})$ and decreased late filling velocity (atrial contraction; A), which are characteristic of failing hearts with elevated LV filling pressure, were observed in MI-saline rats (Figure 3E), whereas in the rAAVS16EPLN-transferred group, the E/A ratio was lowered to near normal (Figure 3F). These and other selected echocardiographic data at 6 months are summarized in Table 2.

Improvement of hemodynamic LV function, assessed by systolic and diastolic LV pressures, contractility, and relaxation, was further confirmed by high-fidelity LV manometry at 6 months after S16EPLN treatment. In the MI-S16EPLN rats, the maximum LV $\mathrm{d} P / \mathrm{d} t$ was significantly higher, and indices of relaxation (minimum $\mathrm{d} P / \mathrm{d} t$ and $\tau$ ) were enhanced compared with those in MI-saline animals (Table 3). Also, the LV end-diastolic pressure (LVEDP), which was considerably elevated in MI-saline rats, was near normal in the S16EPLN treatment group (Table 3). The responses to dobutamine, a $\beta$-adrenergic agonist, are shown in Figure 4. In the MI-saline group, contractility and relaxation measures (maximum and minimum $\mathrm{LV} \mathrm{d} P / \mathrm{d} t$ and $\tau$ ) were severely blunted in response to dobutamine compared with those in the no-MI group. These variables were markedly enhanced by $\beta$-adrenergic stimulation in the MIS16EPLN group compared with those in the MI-saline group, with $\tau$ being near normal at higher dobutamine doses (Figure 4). Estimates of both systolic and diastolic LV wall stress, which were markedly elevated in MI-saline animals, were largely suppressed in the MI-S16EPLN group (Table 3).

Expression of SR $\mathrm{Ca}^{2+}$-handling proteins. The level of S16EPLN expression was estimated as previously described (7). S16EPLN transfer induced a $46 \%$ increase in total immunoreactive PLN (endogenous PLN and S16EPLN) compared with MI-saline. Endogenous PLN was approximately $10 \%$ decreased in S16EPLN-treated animals. The overall relative molecular ratio of the endogenous PLN versus S16EPLN was approximately 1:0.57 in the noninfarcted myocardium from the treatment group. S16EPLN transgene expression over 6 months was confirmed by RT-PCR (Figure 5A). RT-PCR was repeated using purified poly(A) RNA to further confirm that amplification was transcript dependent, and the results were similar (data not shown). The SERCA2 protein level was also estimated by immunoblotting and was by $42 \%$ higher in S16EPLN-treated ani-

\section{Table 2}

Postmortem and echocardiographic analysis at 6 months after S16EPLN gene transfer

\begin{tabular}{|c|c|c|c|}
\hline & No-MI & $\begin{array}{c}\text { After MI } \\
\text { MI-saline }\end{array}$ & MI-S16EPLN \\
\hline Number & 13 & 12 & 12 \\
\hline BW (g) & $646 \pm 21$ & $632 \pm 13$ & $649 \pm 22$ \\
\hline LVW/BW & $1.46 \pm 0.07$ & $1.83 \pm 0.08^{A}$ & $1.56 \pm 0.07^{\mathrm{A}, \mathrm{B}}$ \\
\hline RVW/BW & $0.34 \pm 0.02$ & $0.51 \pm 0.05^{\mathrm{A}}$ & $0.35 \pm 0.02^{A, B}$ \\
\hline LungW/BW & $2.51 \pm 0.09$ & $3.46 \pm 0.48^{\mathrm{A}}$ & $2.58 \pm 0.12^{B}$ \\
\hline Heart rate (bpm) & $290 \pm 9$ & $282 \pm 23$ & $277 \pm 8$ \\
\hline LVEDV (ml) & $0.84 \pm 0.02$ & $1.52 \pm 0.07^{A}$ & $1.28 \pm 0.04^{A, B}$ \\
\hline LVESV (ml) & $0.38 \pm 0.01$ & $1.13 \pm 0.07^{\mathrm{A}}$ & $0.81 \pm 0.03^{A, B}$ \\
\hline LVEF (\%) & $54.7 \pm 1.0$ & $25.8 \pm 1.7^{A}$ & $36.4 \pm 0.8 \mathrm{~A}, \mathrm{~B}$ \\
\hline E/A ratio & $1.67 \pm 0.05$ & $2.32 \pm 0.21^{A}$ & $1.61 \pm 0.05^{B}$ \\
\hline
\end{tabular}

${ }^{A} P<0.05$ vs. no-MI. ${ }^{B} P<0.05$ vs. MI-saline animals. BW, body weight; LVW, LV weight; RVW, right ventricular weight; lungW, lung weight; E/A ratio, Doppler-determined E/A ratio. 
Table 3

Hemodynamic variables at 6 months after S16EPLN gene transfer

\begin{tabular}{lccc}
\hline & \multicolumn{3}{c}{ After MI } \\
& No-MI & MI-saline & MI-S16EPLN \\
Number & 9 & 9 & 8 \\
Heart rate $(\mathrm{bpm})$ & $290 \pm 9$ & $282 \pm 23$ & $277 \pm 8$ \\
PLVP $(\mathrm{mmHg})$ & $114.6 \pm 4.7$ & $105.8 \pm 4.0$ & $114.5 \pm 3.9$ \\
Max dP/d $t(\mathrm{mmHg} / \mathrm{s})$ & $7,949 \pm 268$ & $5,375 \pm 439^{\mathrm{A}}$ & $6,624 \pm 212^{\mathrm{A}}$ \\
Min $\mathrm{d} P / \mathrm{d} t(\mathrm{mmHg} / \mathrm{s})$ & $-6,734 \pm 340$ & $-3,971 \pm 254^{\mathrm{A}}$ & $-5,468 \pm 300^{\mathrm{A}, \mathrm{B}}$ \\
$\tau(\mathrm{ms})$ & $13.7 \pm 0.9$ & $22.1 \pm 1.0^{\mathrm{A}}$ & $17.9 \pm 1.1^{\mathrm{B}}$ \\
LVEDP $(\mathrm{mmHg})$ & $1.8 \pm 0.6$ & $13.4 \pm 1.4^{\mathrm{A}}$ & $3.2 \pm 0.4^{\mathrm{B}}$ \\
Diastolic WS $\left(\mathrm{kdyne} / \mathrm{cm}^{2}\right)$ & $1.05 \pm 0.39$ & $9.20 \pm 1.06^{\mathrm{A}}$ & $2.88 \pm 0.47^{\mathrm{B}}$ \\
Systolic WS $\left(\mathrm{kdyne} / \mathrm{cm}^{2}\right)$ & $24.7 \pm 0.9$ & $59.0 \pm 3.5^{\mathrm{A}}$ & $46.1 \pm 3.9^{\mathrm{A}, \mathrm{B}}$ \\
& & &
\end{tabular}

${ }^{A} P<0.05$ vs. no-MI. ${ }^{B} P<0.05$ vs. MI-saline animals. PLVP, peak LV systolic pressure; max $\mathrm{dP} / \mathrm{d} t$, LV peak rate of change of pressure during isovolumic contraction; min $\mathrm{d} P / \mathrm{d} t$, LV peak rate of change of pressure during isovolumic relaxation; LVEDP, LV end-diastolic pressure; WS, meridional stress of LV wall; kdyne, kilodynes.

mals, most probably reflecting general improvement of cardiac function and suppressed ventricular remodeling.

Suppression of chronic remodeling, fibrosis, and pulmonary effusion in $M I$ animals by S16EPLN treatment. Both the LV/body weight ratio and the right ventricular/body weight ratio were significantly increased in MI-saline rats compared with no-MI rats, and these values were much reduced in MI-S16EPLN rats compared with MI-saline rats (Table 2). The lung/body weight ratio was elevated in the MI-saline group and almost normal in the MI-S16EPLN group (Table 2).

The S16EPLN treatment did not significantly affect changes in the thickness of infarcted LV wall between measurement before gene transfer and measurement 6 months after gene transfer. Changes in the diastolic ventricular anterior wall thickness, measured by M-mode echocardiography, were from $0.95 \pm 0.04 \mathrm{~mm}$ to $0.89 \pm 0.05 \mathrm{~mm}( \pm \mathrm{SE} ; n=12)$ in S16EPLN-treated animals and from $0.98 \pm 0.05 \mathrm{~mm}$ to $0.92 \pm 0.04 \mathrm{~mm}( \pm \mathrm{SE} ; n=12)$ in the salinetreated group. On the other hand, the degree of compensatory cardiomyocyte hypertrophy in the noninfarcted area of myocardium, assessed as cell diameter, was significantly lower in MI-S16EPLN rats than in MI-saline animals (Figure 5E). To test whether the transgene (S16EPLN) affected $\mathrm{Ca}^{2+}$ signaling, which can be linked to cardiomyocyte hypertrophy $(32,33)$ through molecules such as calcium/calmodulin-dependent kinase II (CaMKII) (34) or calcineurin (35), we transfected cultured rat neonatal cardiomyocytes with an adenoviral vector carrying S16EPLN (Adv-S16EPLN) for 36 hours and treated them further with the Gq-coupled agonists phenylephrine and endothelin- 1 for 24 hours. Thereafter, myofibrinogenesis was measured by phalloidin staining and immunostaining with an anti-myosin light chain antibody, and induction of atrial natriuretic peptide (ANP), an embryonic gene marker, was also assessed. S16EPLN overexpression did not induce hypertrophic changes, nor did it alter hypertrophy induced by Gq-coupled agonists in this cell type (data not shown).

The mRNA expression of ANP and skeletal actin was evaluated by Northern blot analysis in the MI rat hearts after gene transfer treatment. Although there was a trend toward suppression of these marker genes in the S16EPLN-treated hearts, quantitative measurement of signal intensity did not reach statistical significance because of specimen-dependent variation in both saline-treated and S16EPLNtreated groups (data not shown).
In quantitative histological analysis, a significantly lower extent of interstitial fibrosis was apparent in the noninfarcted region in the S16EPLN-treated animals versus the MI-saline group (Figure 5F). Histological studies prior to gene transfer were not included in this study, since biopsy would have been required. However, histological evaluation of the noninfarcted $L V$ posterior wall collected from the operated rats in a separate study, which had similar myocardial-infarct sizes, showed only mild signs of fibrosis at 5 weeks after the coronary artery ligation procedure (data not shown).

\section{Discussion}

The findings of this study of a postinfarction rat model with established heart failure demonstrate that in vivo delivery of the S16EPLN gene was highly effective in improving LV function and mitigating adverse remodeling compared with delivery of saline by the same route. LV function, measured by echocardiography, was enhanced in the S16EPLN-treated group, as evidenced by higher LVEF, which was due, at least in part, to reduced afterload (reduced systolic wall stress). In addition, the mutant protein increased contractility, and the positive inotropic effect (increased maximum $L V \mathrm{~d} P / \mathrm{d} t$ ) also was associated with marked improvement in $L V$ relaxation (enhanced minimum $L V \mathrm{~d} P / \mathrm{d} t$ and shortened $\tau)$. LV $\mathrm{d} P / \mathrm{d} t$ is known to be influenced by myocardial contractility and also varies directly with the preload (36), but it is considered to be largely independent of afterload, provided the peak occurs at or before opening of the aortic valve (which was the case in these experiments). Therefore, the finding that this measure was significantly higher in the S16EPLN-treated group than in untreated controls despite lower preload (LVEDP, LVEDV, and diastolic wall stress) indicates that treatment enhanced LV contractility. Diastolic function also was improved by treatment: the E/A ratio was lowered, and LVEDP and diastolic wall stress were nearly normalized. Importantly, unfavorable LV remodeling was largely prevented by rAAV-S16EPLN treatment; this was reflected by limitation of LV dilation and reduction of LV hypertrophy (LV
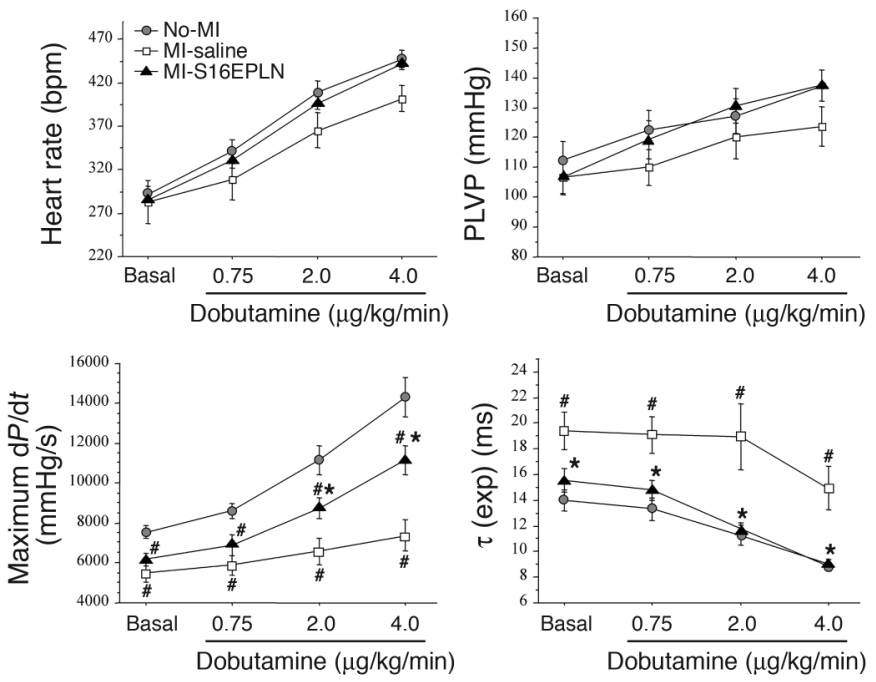

\section{Figure 4}

Responses to $\beta$-adrenergic agonist stimulation in rAAV-S16EPLN-treated versus $\mathrm{Ml}$-saline rats at 6 months after gene transfer. ${ }^{*} P<0.05$, MIS16EPLN vs. MI-saline animals. ${ }^{P} P<0.05$, MI-S16EPLN or MI-saline vs. no-MI animals. PLVP, peak LV systolic pressure. exp, exponential. 


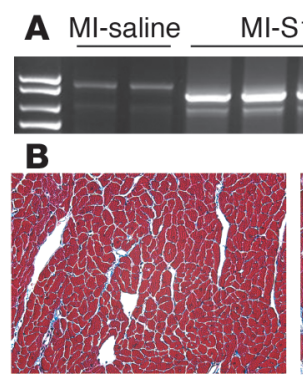

E
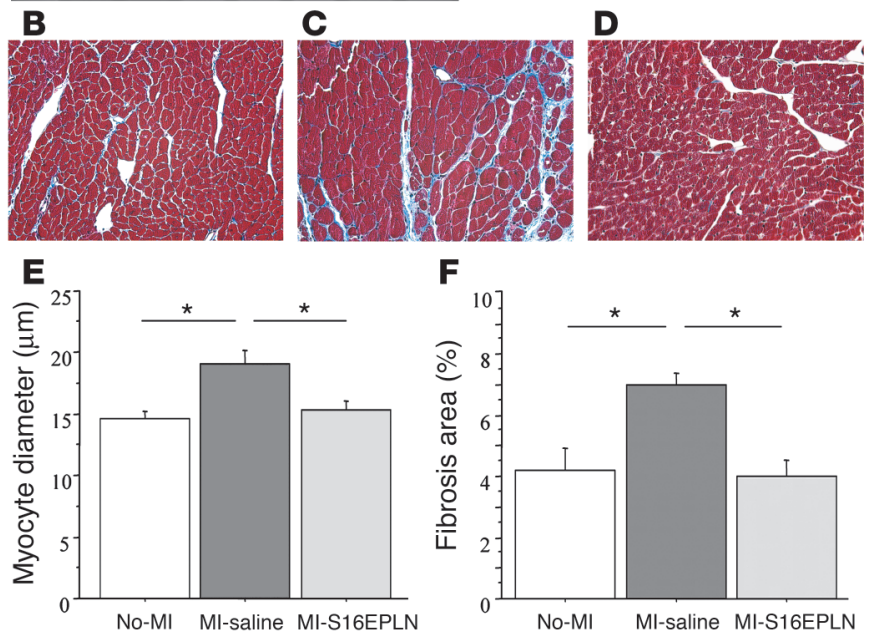

F

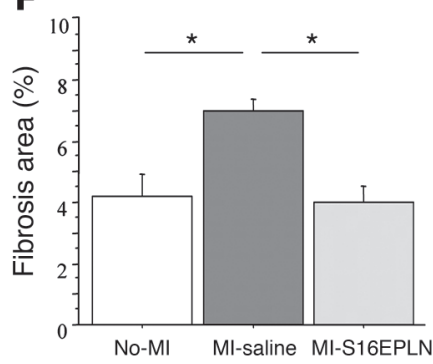

\section{Figure 5}

S16EPLN transgene expression and suppression of histological signs of cardiac remodeling after S16EPLN gene transfer. (A) RT-PCR confirmation of transgene expression in noninfarcted myocardium isolated from S16EPLN-treated hearts at 6 months after gene transfer. Extracted RNA was treated with DNase before RT-PCR. Primers were designed within $5^{\prime}$ noncoding and $3^{\prime}$ noncoding sequences in rAAV-S16EPLN vector, flanking the S16EPLN cDNA coding sequence. (B-D) Representative images of Masson's trichrome staining $(\times 200)$ : no-MI (B), MI-saline (C), and MI-S16EPLN animals (D). (E and F) Myocyte diameters (E) and fibrosis areas $(\mathbf{F})$ were quantitatively analyzed as described in Methods. Studies occurred at 6 months after gene transfer. Data represent mean \pm SE. ${ }^{*} P<0.05$ between groups ( $n=5$ per group). weight and cardiomyocyte size). The right ventricular weight also was significantly lower in the treated group, suggesting that lowered LVEDP led to reduced pulmonary artery and right ventricular pressures (although right heart pressures were not measured). The reduction of the wet lung/body weight ratio with S16EPLN treatment suggests that lowered LVEDP also led to improved pulmonary capillary pressure and reduced lung edema.

We obtained relatively specific cardiac gene expression, even though the CMV promoter is not tissue specific. This can be attributed primarily to the transcoronary delivery of the vector during occlusion of the aorta distal to the site of vector injection (which prevented systemic delivery), and the simultaneous occlusion of the pulmonary artery (which prevented delivery to the lungs). In a previous study using the same gene transfer method in hamsters (7), we observed only occasional cells with minimal $\beta$-gal staining in the liver and ascending aorta, and no cells with $\beta$-gal staining in the brain, liver, spleen, kidney, and lung. We were unable to perform a similar analysis in the present study because of the immune reaction caused by the rAAV-LacZ treatment. In histological examination, we have reevaluated rAAV-LacZ-transferred hamster myocardium, searching for $\beta$-gal-positive staining in the coronary vascular bed and in the perivascular interstitial area, and we observed none. Lack of vascular gene expression after the transcoronary perfusion of rAAV vectors was independently reported by Xiao's group (37). Thus, we speculate that S16EPLN expressed in cardiomyocytes exerted beneficial effects in the failing heart.

Cardiac remodeling after MI includes infarct expansion and eccentric hypertrophy of the noninfarcted left ventricle of the volume-overload type. Eccentric hypertrophy is characterized by progressive chamber dilation and an increase in the ratio of chamber volume to wall thickness (20), a setting that eventually leads to decreased myocardial function and increased myocardial fibrosis (38). Pharmacological approaches in patients with heart failure, such as inhibition of angiotensin-converting enzyme and use of ventricular-assist devices, have demonstrated that remodeling can be a partly reversible process (so-called reverse remodeling) (39); ventricular-assist devices can produce especially marked reverse modeling (40). In the current study, the randomization and gene transfer were performed at 5 weeks after coro- nary occlusion, when expansion and scarring of the infarct are nearly complete (41). The infarct size was closely comparable at randomization in S16EPLN-treated and control saline-treated groups, and the success of the randomization is further indicated by the closely comparable echocardiographic measurements of LV size and function in the two groups before the gene transfer procedure. On the other hand, postmortem infarct size was not an endpoint measure of this study, since infarct size is expressed relative to the size of the left ventricle, and we expected, and found, substantial differences in remodeling of the surviving myocardium between treated and untreated groups.

It is of interest that the favorable global actions of the S16EPLN gene in the heart occurred despite a limited transcoronary transduction efficiency, which is estimated at $60 \%$ or less of the cell population in the noninfarcted myocardium, based on the initial LacZ expression studies. We attribute these effects on global function, including enhanced $\beta$-adrenergic responses found on hemodynamic analysis, in part to reduced loading on the left ventricle in the S16EPLN-treated group, indicated by reduction of the estimated end-systolic and end-diastolic wall-stress values. A decrease in wall stress has favorable effects on remodeling of noninfarcted remote myocardium, a process that may involve apoptotic cell loss $(42,43)$. It is also possible that lowered wall stress, particularly in diastole, could have reduced subendocardial ischemia, if present, by improving myocardial blood flow to the inner LV wall, thereby improving cardiac function (44). In a study of sustained aortic stenosis in PLNnull mice by Kiriazis et al. (15), a relatively greater pressure gradient across the banding might have contributed to the trend of greater incidence of heart failure in that group of mice. On the other hand, in the MLP cardiomyopathic mouse model, evidence suggested that wall-stress reduction, which was achieved by PLN ablation, prevents development of heart failure by maintaining structural and functional integrity of the Z-disc/titin complex (45). Abnormalities of this complex also may occur in a recently identified subset of patients with dilated cardiomyopathy who harbor mutations in the telethonin-interacting domain of MLP, telethonin being a titinbinding molecule (45). We postulate that S16EPLN-treated MI rats had more favorable loading on all the cells, both S16EPLN-transfected and nontransfected cardiomyocytes, in the noninfarcted 
region of the left ventricle, and that systolic unloading due to enhanced contractility of transfected cells contributed to a reduction of end-systolic volume and a higher ejection fraction. Diastolic unloading due to improved relaxation and lower end-diastolic LV volume contributed to enhanced diastolic function and reduction of unfavorable remodeling.

In a recent report, the effects of PLN ablation, produced by crossbreeding with PLN-null mice, were assessed in two genetic mouse models, G $\alpha$ q overexpression and targeted mutation of myosin-binding protein $\mathrm{C}$, both of which exhibited extensive cardiomyopathic changes associated with the development of cardiac dysfunction and dilation (14). No effect of PLN ablation on echocardiographic measures of hypertrophy and dysfunction were observed in either model, and impaired hemodynamic responsiveness to $\beta$-adrenergic stimulation was not affected (14). These findings contrast with those of our previous study on PLN ablation in MLP cardiomyopathic mice (using a similar crossbreeding approach), in which the cardiomyopathic phenotype did not develop (12), and they also differ from the present findings in the adult rat with $\mathrm{MI}$, in which an entirely different approach was used to achieve PLN inhibition in the presence of established cardiac dysfunction and dilation.

It seems possible that in the two transgenic models described above (14), the strong genetic stimuli mediating cardiomyopathic changes, present in utero and thereafter, may have overshadowed the effect of PLN ablation to enhance cardiomyocyte contractility. In the MLP cardiomyopathic model, on the other hand, cardiac dilation and hypertrophy develop progressively over several weeks after birth $(45,46)$, and in that setting, correction by PLN ablation of the $\mathrm{Ca}^{2+}$ handling defect during postnatal cardiac growth may explain the observed complete prevention of the dilated cardiomyopathy phenotype (12). Thus, we postulate that enhanced cardiac function maintained normal heart size, obviating the progressive cardiac dilation associated with increased wall stress and secondary cardiac hypertrophy present in MLP-null mice (47).

Recently, an R9C mutation of PLN was identified in patients with familial cardiomyopathy (16), and suppression of Ser16 phosphorylation of wild-type PLN was proposed as the mechanism of the dominant negative effect of this mutation (16). The R9C PLN itself also lacks the ability to phosphorylate Ser16. This finding supports our concept that overexpression of the pseudophosphorylated form of PLN (S16EPLN) has the opposite effect, leading to a chronic beneficial effect on the failing myocardium. On the other hand, in another recent study, an L39stop mutation in PLN in patients with hereditary cardiomyopathy was reported to represent a possible long-term adverse effect of innate absence of PLN (17). The precise role of the L39stop mutation in humans remains to be clarified, since the unstable truncated L39stop PLN peptide contains a portion of the cytoplasmic inhibitor domain of PLN that could inhibit SERCA2 (47).

PLN is an endogenous muscle-specific inhibitor of SERCA2, and the regulation of its inhibitory function is mainly via its phosphorylation by cAMP-dependent protein kinase at Ser16, and also by CaMKII (48) at Thr17. Cardiac-specific disruption of PLN in mice results in a marked increase in myocardial contractility, without change in the maximum cardiac response to $\beta$-adrenergic stimulation (48). CaMKII-dependent phosphorylation of PLN may contribute to a positive force-frequency relationship and to frequencydependent acceleration of relaxation (49), although Bers's group has recently reported that the latter event might be at least partly independent of PLN (50). As potential therapeutic strategies for heart failure, two approaches have been employed to enhance the reuptake of $\mathrm{Ca}^{2+}$ into the cardiac SR through this mechanism: alteration of the PLN/SERCA2 molecular ratio, and reduction of PLN activity. These approaches have included gene transfer of SERCA2 $(10,51)$, use of antisense PLN RNA (52-54), a single peptide antibody designed against PLN (55), and overexpression of mutant forms of PLN to interfere with the interaction of SERCA2 and endogenous $\operatorname{PLN}(7,12,53)$ - an approach we initially used in cardiomyopathic hamsters in vivo (7). In our study in cardiomyopathic hamsters, however, the rapid deterioration of function, myocyte loss, and increasing fibrosis that occur consequent to the absence of $\delta$-sarcoglycan in this genetic form of cardiomyopathy $(21,56)$ limited longterm beneficial effects. Therefore, in the present study, we applied rAAV-S16EPLN gene therapy in the rat post-MI model of chronic heart failure, in which sustained overload on noninfarcted regions is the major factor in the chronic progression of heart failure.

Our finding that $\beta$-adrenergic responsiveness to dobutamine was well maintained after gene transfer of S16EPLN may reflect the less marked heart failure in the treated group. This finding suggests that such treatment could enhance effects of sympathetic neurohormonal activation during exercise to increase cardiac function, acting in part by amplification of the force-frequency effect produced by $\beta$-adrenergic stimulation, which is reduced or absent in heart failure (57). Long-term inhibition of PLN also might lead to more effective adjunctive use of other therapies.

The single-stranded DNA virus vector $\mathrm{rAAV}$ is widely regarded as nonpathogenic and can achieve long-term gene expression in a variety of cell types, including myocytes $(7,58)$. The reason for the marked late cardiac inflammatory reaction to rAAV-LacZ and associated low efficiency of gene expression in rats after 5 weeks of gene transfer is not completely clear. Different preparations of rAAV-LacZ and an rAAV vector carrying an enhanced GFP reproducibly provoked similar tissue reactions, and we also found the reaction to occur in a different subspecies of rat (data not shown). The virus itself did not appear to be involved, since no signs of inflammatory reaction were evident in the rAAV-S16EPLN-treated group over 6 months. Therefore, we suspect that an immunoreaction to LacZ and enhanced GFP, which are completely foreign proteins to rats, was responsible, since S16EPLN is a minimally modified peptide derived from the native human PLN. Others have described high local efficiency of rAAV-LacZ transduction at 8 weeks in rats after direct intramyocardial injection, with no report of an immune response to LacZ (59). On the other hand, immunoreaction to foreign genes, such as LacZ, can occur in mice under certain circumstances (60). Species differences in immunoreactivity to rAAV-LacZ are suggested by our finding of no immunogenic reaction in a reexamination of cardiomyopathic hamster hearts in our previous study (7).

We consider that rAAV-S16EPLN could be an ideal vector for therapeutic use (5), not only because of its beneficial physiological effects and long-term action, but also because of its minimal modification from native PLN. It also has the advantage that LV contraction is enhanced in the absence of $\beta$-adrenergic receptor stimulation at rest, and without the sustained increase of intracellular cAMP that is associated with the chronic use of most positive inotropic agents, which have generally produced deleterious effects including increased mortality in patients with chronic heart failure (61). In this regard, ryanodine receptor phosphorylation induced by cAMP-dependent kinase in failing hearts may cause diastolic SR $\mathrm{Ca}^{2+}$ leakage, and associated malignant arrhythmia (62). The present study was powered in terms of numbers of rats to detect significant difference in cardiac function 
and remodeling between groups at 6 months, followed by a terminal hemodynamic study, so that longer follow-up is not currently available. The degree of LV dilation may be the most powerful predictor of long-term prognosis in patients after MI (63), and additional studies on rats with heart failure after MI using larger numbers of animals and longer follow-up should clarify the effects of PLN inhibition on arrhythmias and survival. One animal died in the S16EPLN-treated group without evidence of heart failure. Although the difference in mortality was significant by preliminary Kaplan-Meier analysis, the numbers are inadequate to prove an effect of PLN inhibition in a formal survival analysis. S16EPLN-treated animals did not develop ventricular premature contractions either during repeated echocardiographic observations or during the final hemodynamic studies with intravenous catecholamine administration. Nevertheless, we recognize the possibility that S16EPLN treatment might induce arrhythmias in longer-term studies.

Our findings of enhanced function, reduced remodeling and fibrosis, and lessened heart failure in an animal model relevant to the setting of heart failure in patients after a large acute MI lend support to the potential therapeutic usefulness of this gene transfer approach. In addition, these studies document that the therapeutic effects of PLN inhibition can be extended to acquired forms of heart failure, suggesting that previous results in some genetic models ( 7 , 12) may be generalizable.

\section{Acknowledgments}

We acknowledge partial fellowship support for Y. Iwanaga from $\mathrm{NicO}$ SA and for T. Dieterle from the Swiss National Science Foundation and the Freiwillige Akademische Gesellschaft (Basel, Switzerland). General support was provided by the Leducq Foundation and by grant HL53773 from the National Heart, Lung, and Blood Institute.

Received for publication May 20, 2003, and accepted in revised form December 28, 2003.

Address correspondence to: John Ross, Jr., Department of Medicine, University of California, San Diego, 9500 Gilman Drive, La Jolla, California 92093-0613B, USA. Phone: (858) 822-2267; Fax: (858) 5341626; E-mail: jross@ucsd.edu.

Yoshitaka Iwanaga's present address is: Research Institute, National Cardiovascular Center, Osaka, Japan.
1. Cohn, J.N., et al. 1997. Report of the National Heart, Lung, and Blood Institute Special Emphasis Panel on Heart Failure Research. Circulation. 95:766-770.

2. Hosenpud, J.D., Bennett, L.E., Keck, B.M., Boucek, M.M., and Novick, R.J. 2001. The Registry of the International Society for Heart and Lung Transplantation: eighteenth Official Report. J. Heart Lung Transplant. 20:805-815.

3. Hoshijima, M., and Chien, K.R. 2002. Mixed signals in heart failure: cancer rules. J. Clin. Invest. 109:849-855. doi:10.1172/JCI200215380.

4. Chien, K.R. 1999. Stress pathways and heart failure. Cell. 98:555-558.

5. Somia, N., and Verma, I.M. 2000. Gene therapy: trials and tribulations. Nat. Rev. Genet. 1:91-99.

6. Ikeda, Y., et al. 2002. Restoration of deficient membrane proteins in the cardiomyopathic hamster by in vivo cardiac gene transfer. Circulation. 105:502-508.

7. Hoshijima, M., et al. 2002. Chronic suppression of heart-failure progression by a pseudophosphorylated mutant of phospholamban via in vivo cardiac rAAV gene delivery. Nat. Med. 8:864-871.

8. Marx, S.O., et al. 2000. PKA phosphorylation dissociates FKBP12.6 from the calcium release channel (ryanodine receptor): defective regulation in failing hearts. Cell. 101:365-376.

9. Morgan, J.P., Erny, R.E., Allen, P.D., Grossman, W., and Gwathmey, J.K. 1990. Abnormal intracellular calcium handling, a major cause of systolic and diastolic dysfunction in ventricular myocardium from patients with heart failure. Circulation. 81:III21-III32.

10. del Monte, F., et al. 2001. Improvement in survival and cardiac metabolism after gene transfer of sarcoplasmic reticulum $\mathrm{Ca}(2+)$-ATPase in a rat model of heart failure. Circulation. 104:1424-1429.

11. Shah, A.S., et al. 2001. In vivo ventricular gene delivery of a beta-adrenergic receptor kinase inhibitor to the failing heart reverses cardiac dysfunction. Circulation. 103:1311-1316.

12. Minamisawa, S., et al. 1999. Chronic phospholamban-sarcoplasmic reticulum calcium ATPase interaction is the critical calcium cycling defect in dilated cardiomyopathy. Cell. 99:313-322.

13. Freeman, K., et al. 2001. Alterations in cardiac adrenergic signaling and calcium cycling differentially affect the progression of cardiomyopathy. J. Clin. Invest. 107:967-974.

14. Song, Q., et al. 2003. Rescue of cardiomyocyte dysfunction by phospholamban ablation does not prevent ventricular failure in genetic hypertrophy. J. Clin.
Invest. 111:859-867. doi:10.1172/JCI200316738.

15. Kiriazis, H., et al. 2002. Hypertrophy and functional alterations in hyperdynamic phospholambanknockout mouse hearts under chronic aortic stenosis. Cardiovasc. Res. 53:372-381.

16. Schmitt, J.P., et al. 2003. Dilated cardiomyopathy and heart failure caused by a mutation in phospholamban. Science. 299:1410-1413.

17. Haghighi, K., et al. 2003. Human phospholamban null results in lethal dilated cardiomyopathy revealing a critical difference between mouse and human. J. Clin. Invest. 111:869-876. doi:10.1172/JCI200317892.

18. Xiao, X., Li, J., and Samulski, R.J. 1998. Production of high-titer recombinant adeno-associated virus vectors in the absence of helper adenovirus. J. Virol. 72:2224-2232.

19. Auricchio, A., Hildinger, M., O'Connor, E., Gao, G.P., and Wilson, J.M. 2001. Isolation of highly infectious and pure adeno-associated virus type 2 vectors with a single-step gravity-flow column. Hum. Gene Ther. 12:71-76.

20. Hongo, M., et al. 1998. Angiotensin II blockade followed by growth hormone as adjunctive therapy after experimental myocardial infarction. J. Card. Fail. 4:213-224.

21. Ryoke, T., et al. 1999. Progressive cardiac dysfunction and fibrosis in the cardiomyopathic hamster and effects of growth hormone and angiotensin-converting enzyme inhibition. Circulation. 100:1734-1743.

22. Schiller, N.B., et al. 1989. Recommendations for quantitation of the left ventricle by two-dimensional echocardiography. American Society of Echocardiography Committee on Standards, Subcommittee on Quantitation of Two-Dimensional Echocardiograms. J. Am. Soc. Echocardiogr. 2:358-367.

23. Collins, K.A., Korcarz, C.E., and Lang, R.M. 2003. Use of echocardiography for the phenotypic assessment of genetically altered mice. Physiol. Genomics. 13:227-239.

24. Sheehan, F.H., et al. 1986. Advantages and applications of the centerline method for characterizing regional ventricular function. Circulation. 74:293-305.

25. Litwin, S.E., Katz, S.E., Morgan, J.P., and Douglas, P.S. 1994. Serial echocardiographic assessment of left ventricular geometry and function after large myocardial infarction in the rat. Circulation. 89:345-354

26. Baluk, P., et al. 1997. Endothelial gaps: time course of formation and closure in inflamed venules of rats. Am. J. Physiol. 272:L155-L170.
27. Tanaka, M., et al. 1986. Quantitative analysis of myocardial fibrosis in normals, hypertensive hearts, and hypertrophic cardiomyopathy. Br. Heart J. 55:575-581.

28. Inada, T., et al. 1999. Upregulated expression of cardiac endothelin-1 participates in myocardial cell growth in Bio14.6 Syrian cardiomyopathic hamsters. J. Am. Coll. Cardiol. 33:565-571.

29. Frank, K., Tilgmann, C., Shannon, T.R., Bers, D.M., and Kranias, E.G. 2000. Regulatory role of phospholamban in the efficiency of cardiac sarcoplasmic reticulum Ca2+ transport. Biochemistry. 39:14176-14182.

30. Zhou, Y.Y., et al. 2000. Culture and adenoviral infection of adult mouse cardiac myocytes: methods for cellular genetic physiology. Am. J. Physiol. Heart Circ. Physiol. 279:H429-H436.

31. Hajjar, R.J., Schmidt, U., Kang, J.X., Matsui, T., and Rosenzweig, A. 1997. Adenoviral gene transfer of phospholamban in isolated rat cardiomyocytes. Rescue effects by concomitant gene transfer of sarcoplasmic reticulum $\mathrm{Ca}(2+)$-ATPase. Circ. Res. 81:145-153.

32. Molkentin, J.D., and Dorn, I.G., 2nd. 2001. Cytoplasmic signaling pathways that regulate cardiac hypertrophy. Annu. Rev. Physiol. 63:391-426.

33. Frey, N., McKinsey, T.A., and Olson, E.N. 2000. Decoding calcium signals involved in cardiac growth and function. Nat. Med. 6:1221-1227.

34. Ramirez, M.T., Zhao, X.L., Schulman, H., and Brown, J.H. 1997. The nuclear deltaB isoform of $\mathrm{Ca} 2+/$ calmodulin-dependent protein kinase II regulates atrial natriuretic factor gene expression in ventricular myocytes. J. Biol. Chem. 272:31203-31208.

35. Molkentin, J.D., et al. 1998. A calcineurin-dependent transcriptional pathway for cardiac hypertrophy. Cell. 93:215-228.

36. Little, W.C. 1985. The left ventricular dP/dtmax-enddiastolic volume relation in closed-chest dogs. Circ. Res. 56:808-815.

37. Li, J., et al. 2003. Efficient and long-term intracardiac gene transfer in delta-sarcoglycan-deficiency hamster by adeno-associated virus- 2 vectors. Gene Ther. 10:1807-1813.

38. Swynghedauw, B. 1999. Molecular mechanisms of myocardial remodeling. Physiol. Rev. 79:215-262.

39. Hayashida, W., Van Eyll, C., Rousseau, M.F., and Pouleur, H. 1993. Regional remodeling and nonuniform changes in diastolic function in patients with left ventricular dysfunction: modification by longterm enalapril treatment. The SOLVD Investigators. 
J. Am. Coll. Cardiol. 22:1403-1410.

40. Zafeiridis, A., Jeevanandam, V., Houser, S.R., and Margulies, K.B. 1998. Regression of cellular hypertrophy after left ventricular assist device support. Circulation. 98:656-662.

41. Mukherjee, R., et al. 2003. Myocardial infarct expansion and matrix metalloproteinase inhibition. Circulation. 107:618-625.

42. Nadal-Ginard, B., Kajstura, J., Leri, A., and Anversa, P. 2003. Myocyte death, growth, and regeneration in cardiac hypertrophy and failure. Circ. Res. 92:139-150.

43. Mani, K., and Kitsis, R.N. 2003. Myocyte apoptosis: programming ventricular remodeling. J. Am. Coll. Cardiol. 41:761-764.

44. Ross, J., Jr. 1991. Myocardial perfusion-contraction matching. Implications for coronary heart disease and hibernation. Circulation. 83:1076-1083.

45. Knoll, R., et al. 2002. The cardiac mechanical stretch sensor machinery involves a $Z$ disc complex that is defective in a subset of human dilated cardiomyopathy. Cell. 111:943-955.

46. Hongo, M., et al. 2000. Effects of growth hormone on cardiac dysfunction and gene expression in genetic murine dilated cardiomyopathy. Basic Res. Cardiol. 95:431-441.

47. Sasaki, T., Inui, M., Kimura, Y., Kuzuya, T., and Tada, M. 1992. Molecular mechanism of regulation of $\mathrm{Ca} 2+$ pump ATPase by phospholamban in cardiac sarcoplasmic reticulum. Effects of synthetic phospholamban peptides on Ca2+ pump ATPase. J. Biol.
Chem. 267:1674-1679.

48. Brittsan, A.G., and Kranias, E.G. 2000. Phospholamban and cardiac contractile function. J. Mol. Cell. Cardiol. 32:2131-2139.

49. Hagemann, D., and Xiao, R.P. 2002. Dual site phospholamban phosphorylation and its physiological relevance in the heart. Trends Cardiovasc. Med. 12:51-56.

50. DeSantiago, J., Maier, L.S., and Bers, D.M. 2002. Frequency-dependent acceleration of relaxation in the heart depends on CaMKII, but not phospholamban. J. Mol. Cell. Cardiol. 34:975-984.

51. Miyamoto, M.I., et al. 2000. Adenoviral gene transfer of SERCA2a improves left-ventricular function in aortic-banded rats in transition to heart failure. Proc. Natl. Acad. Sci. U. S. A. 97:793-798.

52. Eizema, K., et al. 2000. Adenovirus-based phospholamban antisense expression as a novel approach to improve cardiac contractile dysfunction: comparison of a constitutive viral versus an endothelin-1-responsive cardiac promoter. Circulation. 101:2193-2199.

53. He, H., et al. 1999. Effects of mutant and antisense RNA of phospholamban on SR $\mathrm{Ca}(2+)$-ATPase activity and cardiac myocyte contractility. Circulation. 100:974-980.

54. del Monte, F., Harding, S.E., Dec, G.W., Gwathmey, J.K., and Hajjar, R.J. 2002. Targeting phospholamban by gene transfer in human heart failure. Circulation. 105:904-907.

55. Dieterle, T., et al. 2003. Gene transfer of contractilin, a recombinant inotropic antibody-based protein, improves cardiac function in the BIO 14.6 hamster. J. Am. Coll. Cardiol. 41(Suppl. A):217A. (Abstr.)

56. Ikeda, Y., et al. 2000. Altered membrane proteins and permeability correlate with cardiac dysfunction in cardiomyopathic hamsters. Am. J. Physiol. Heart Circ. Physiol. 278:H1362-H1370.

57. Ross, J., Jr. 1998. Adrenergic regulation of the forcefrequency effect. Basic Res. Cardiol. 93:95-101.

58. Smith-Arica, J.R., and Bartlett, J.S. 2001. Gene therapy: recombinant adeno-associated virus vectors. Curr. Cardiol. Rep. 3:43-49.

59. Melo, L.G., et al. 2002. Gene therapy strategy for long-term myocardial protection using adeno-associated virus-mediated delivery of heme oxygenase gene. Circulation. 105:602-607.

60. Brockstedt, D.G., et al. 1999. Induction of immunity to antigens expressed by recombinant adeno-associated virus depends on the route of administration. Clin. Immunol. 92:67-75.

61. Stevenson, L.W. 1998. Inotropic therapy for heart failure. N. Engl. J. Med. 339:1848-1850.

62. Marks, A.R., Priori, S., Memmi, M., Kontula, K., and Laitinen, P.J. 2002. Involvement of the cardiac ryanodine receptor/calcium release channel in catecholaminergic polymorphic ventricular tachycardia. J. Cell. Physiol. 190:1-6.

63. Picard, M.H., Wilkins, G.T., Ray, P.A., and Weyman, A.E. 1990. Natural history of left ventricular size and function after acute myocardial infarction. Assessment and prediction by echocardiographic endocardial surface mapping. Circulation. 82:484-494. 